\title{
ON CERTAIN HESSENBERG MATRICES RELATED WITH LINEAR RECURRENCES
}

\author{
Sibel Koparal, Neşe Ömür and Cemile D. Çolak
}

\begin{abstract}
In this paper, we present various results for permanents and determinants of some Hessenberg matrices. Also, some special cases for permanents are given.
\end{abstract}

Keywords: Hessenberg matrices, permanents, determinants.

\section{Introduction}

Matrix methods are useful tools deriving some properties of linear recurrences. Some authors obtained many connections between certain sequences and permanents of Hessenberg matrices in the literature [1]-[4],[6],[10]-[12].

The permanent of an $n$ - square matrix $\mathbf{A}_{\mathbf{n}}=\left[a_{i j}\right]$ is defined by

$$
\operatorname{per} \mathbf{A}_{\mathbf{n}}=\sum_{\sigma \in D_{n}} \prod_{i=1}^{n} a_{i \sigma(i)}
$$

where the summation extends over all permutations $\sigma$ of the symmetric group $D_{n}$.

In [9], Minc defined the super-diagonal matrix and showed that the permanent of the matrix equals the order k-Fibonacci number.

In [5], Kılıç derived recurrence relations and generating matrices for the sums of usual tribonacci numbers and $4 n$ subscripted tribonacci sequences $\left\{T_{4 n}\right\}$, and their sums. Also, the relationships between these sequences and permanents of certain matrices are obtained.

In [6], Kılıç and Taşcı found the relationships between the sums of the Fibonacci and Lucas numbers and 1-factors of bipartite graphs.

In [7], Kılıç and Taşcı defined the $n \times n$ tridiagonal Toeplitz $(0,-1,1)$-matrix $\mathbf{M}_{\mathbf{n}}=\left[m_{i, j}\right]$ with $m_{i, i}=-1$ for $1 \leq i \leq n, m_{i, i+1}=m_{i+1, i}=1$ for $1 \leq i \leq n-1$

Received November 16, 2017; accepted February 23, 2018

2010 Mathematics Subject Classification. Primary 11B37; Secondary 05A15 
and 0 otherwise, and the $n \times n$ tridiagonal Toeplitz $(0,-1,1)$-matrix $\mathbf{L}_{\mathbf{n}}=\left[l_{i, j}\right]$ with $l_{i, i}=-1$ for $2 \leq i \leq n, l_{i, i+1}=l_{i+1, i}=1$ for $1 \leq i \leq n-1, l_{1,1}=-\frac{1}{2}$ and 0 otherwise. They showed $\operatorname{per} \mathbf{M}_{\mathbf{n}}=F_{-(n+1)}$ and $\operatorname{per} \mathbf{L}_{\mathbf{n}}=\frac{L_{-n}}{2}$, where $F_{n}$ and $L_{n}$ is the $n$th Fibonacci and Lucas numbers, respectively.

In [8], Li showed new Fibonacci-Hessenberg matrices and gave another proof of the well-known results relative to the Pell and Perrin numbers.

In [3], Kalman showed that the $(n+k)$-th term of a sequence is defined recursively as a linear combination of the preceding $k$ terms:

$$
u_{n+k}=c_{0} u_{n}+c_{1} u_{n+1}+\ldots+c_{k-1} u_{n+k-1}
$$

in which the initial terms $u_{0}=\ldots=u_{k-2}=0, u_{k-1}=1$ and $c_{0}, c_{1}, \ldots, c_{k-1}$ are constants.

In [10], considering the generalized Fibonacci-Narayana sequence $\left\{G_{n}(a, c, r)\right\}$, Ramírez derived some relations between this sequence and a permanent of one type of the upper Hessenberg matrix. For example,

$$
\operatorname{per}\left[\begin{array}{cccccccc}
a & c & c & \cdots & c & & & 0 \\
1 & a & 0 & 0 & \cdots & c & & \\
& \ddots & \ddots & \ddots & \ddots & & \ddots & \\
& & 1 & a & 0 & 0 & \cdots & c \\
& & & \ddots & \ddots & \ddots & \ddots & \\
& & & & 1 & a & 0 & 0 \\
& & & & & 1 & a & 0 \\
& & & & & & 1 & a
\end{array}\right]=G_{n+r-1}(a, c, r)
$$

where the generalized Fibonacci-Narayana sequence $\left\{G_{n}(a, c, r)\right\}_{n \in \mathbb{N}}$ is defined as follows:

$$
G_{n}(a, c, r)=a G_{n-1}(a, c, r)+c G_{n-r}(a, c, r), 2 \leq r \leq n,
$$

with the initial conditions $G_{0}(a, c, r)=0, G_{i}(a, c, r)=1$, for $i=1,2, \ldots, r-1$.

In [12], Trojovský defined tridiagonal matrices $\mathbf{B}_{\mathbf{n}}^{\delta}=\left[b_{i j}^{\delta}\right]$ in the form

$$
\left\{\begin{array}{cc}
1 & \text { if } i=j \text { or } i=j-1, \\
(-1)^{j+\delta} & \text { if } i=j+1, \\
0 & \text { otherwise, }
\end{array}\right.
$$

where $\delta \in\{0,1\}$ and showed

$$
\operatorname{det} \mathbf{B}_{\mathbf{n}}^{\delta}=\left\{\begin{array}{ccc}
F_{(n+4-6 \delta) / 2} & \text { if } n \equiv 0 \quad(\bmod 2), \\
F_{(n+1) / 2} & \text { if } n \equiv 1 \quad(\bmod 2) .
\end{array}\right.
$$

\section{Some results}

In this section, we define the sequence $\left\{R_{n}(a, b, c, d)\right\}$ and determine some relationships between the terms of this sequence and permanents of certain upper Hessen- 
berg matrices. A sequence $\left\{R_{n}(a, b, c, d)\right\}$ is defined by for $3 \leq d \leq n$,

$$
R_{n}(a, b, c, d)=a R_{n-1}(a, b, c, d)+b R_{n-2}(a, b, c, d)+c R_{n-d}(a, b, c, d),
$$

in which $R_{0}(a, b, c, d)=0, R_{1}(a, b, c, d)=R_{2}(a, b, c, d)=\ldots=R_{d-2}(a, b, c, d)=1$ and $R_{d-1}(a, b, c, d)=a$. The sequence $\left\{R_{n}(a, b, c, d)\right\}$ is a generalization of the tribonacci sequence. When $a=b=c=1$ and $d=3, R_{n}(1,1,1,3)=T_{n}$ (the $n$th tribonacci number). If $c=0$ and $d=3$, the generalized Fibonacci sequence $\left\{U_{n}(a, b)\right\}$ is obtained. If $a=b=1, c=0$ and $d=3$, the Fibonacci sequence $\left\{F_{n}\right\}$ is obtained and if $a=c=1, b=0$ and $d=3$, the Narayana sequence is obtained.

The generating function $R(z)$ of $R_{n}(a, b, c, d)$ is given by

$$
R(z)=\frac{(a-1+b z) z^{d-1}-b z^{3}-a z^{2}+z}{(1-z)\left(1-a z-b z^{2}-c z^{d}\right)} .
$$

Now we give relationships between terms of the sequence $\left\{R_{n}(a, b, c, d)\right\}$ and the permanents of certain matrices.

For $n \geq 1$, define a $n \times n$ matrix $\mathbf{H}_{\mathbf{n}}(a, b, c, d, k, t)=\left[h_{i, j}\right]$ with $h_{i+1, i}=1$ for $1 \leq i \leq n-2, h_{i, i}=a$ for $1 \leq i \leq n-1, h_{i, i+1}=b$ for $1 \leq i \leq n-1, h_{1, i}=c$ for $3 \leq i \leq d, h_{i, d+i-1}=c$ for $2 \leq i \leq n-d+1, h_{n, n-1}=k, h_{n, n}=t$, and 0 otherwise, i.e.,

$$
\mathbf{H}_{\mathbf{n}}(a, b, c, d, k, t)=\left[\begin{array}{cccccccc}
a & b & c & \cdots & c & 0 & \ldots & 0 \\
1 & a & b & 0 & \cdots & c & \ldots & \vdots \\
& \ddots & \ddots & \ddots & \ddots & \ldots & \ddots & 0 \\
& & 1 & a & b & 0 & \cdots & c \\
& & & \ddots & \ddots & \ddots & \ddots & \ddots \\
& & & & \ddots & \ddots & \ddots & 0 \\
& & & & & 1 & a & b \\
0 & & & & & & k & t
\end{array}\right] .
$$

Then we give the following Theorem.

Theorem 2.1. Let $\mathbf{H}_{\mathbf{n}}(a, b, c, d, k, t)$ be the matrix defined in (2.2). Then, for $n \geqslant 1$ and $d \geq 3$,

$$
\operatorname{per} \mathbf{H}_{\mathbf{n}}(a, b, c, d, k, t)=k R_{n+d-2}(a, b, c, d)-(k a-t) R_{n+d-3}(a, b, c, d),
$$

where the real numbers $k$ and $t$.

Proof. (Induction on $n$ ) If $n=1$, then we have

$$
\operatorname{per} \mathbf{H}_{\mathbf{1}}(a, b, c, d, k, t)=t=k R_{d-1}(a, b, c, d)-(k a-t) R_{d-2}(a, b, c, d) .
$$


Suppose that the equation holds for $n-1$. Then we show that the equation holds for $n$. Expanding the $\operatorname{per} \mathbf{H}_{\mathbf{n}}$ with respect to the last column $d$ times, we write

$$
\begin{aligned}
& \operatorname{per} \mathbf{H}_{\mathbf{n}}(a, b, c, d, k, t) \\
= & \operatorname{aper} \mathbf{H}_{\mathbf{n}-\mathbf{1}}(a, b, c, d, k, t)+\operatorname{bper} \mathbf{H}_{\mathbf{n}-\mathbf{2}}(a, b, c, d, k, t)+\operatorname{cper} \mathbf{H}_{\mathbf{n}-\mathbf{d}}(a, b, c, d, k, t) .
\end{aligned}
$$

By our assumption, we have

$$
\begin{aligned}
\operatorname{per}_{\mathbf{n}}(a, b, c, d, k, t)= & a\left(k R_{n+d-3}(a, b, c, d)-(k a-t) R_{n+d-4}(a, b, c, d)\right) \\
& +b\left(k R_{n+d-4}(a, b, c, d)-(k a-t) R_{n+d-5}(a, b, c, d)\right) \\
& +c\left(k R_{n-2}(a, b, c, d)-(k a-t) R_{n-3}(a, b, c, d)\right) \\
= & k R_{n+d-2}(a, b, c, d)-(k a-t) R_{n+d-3}(a, b, c, d) .
\end{aligned}
$$

Thus, the proof is complete.

When $t=a$ and $k=1$ in (2.3), we have $\operatorname{per}_{\mathbf{n}}(a, b, c, d, 1, a)=R_{n+d-2}(a, b, c, d)$.

For $n \geq 1$, define a $n \times n$ matrix $\mathbf{E}_{\mathbf{n}}(a, b, c, d, k, t)=\left[e_{i, j}\right]$ with $e_{i+1, i}=-1$ for $1 \leq i \leq n-2, e_{i, i}=a$ for $1 \leq i \leq n-1, e_{i, i+1}=b$ for $1 \leq i \leq n-1, e_{1, i}=c$ for $3 \leq i \leq d, e_{i, d+i-1}=c$ for $2 \leq i \leq n-d+1, e_{n, n-1}=-k, e_{n, n}=t$, and 0 otherwise, i.e.,

$$
\mathbf{E}_{\mathbf{n}}(a, b, c, d, k, t)=\left[\begin{array}{cccccccc}
a & b & c & \cdots & c & 0 & \ldots & 0 \\
-1 & a & b & 0 & \ldots & c & \ldots & \vdots \\
& \ddots & \ddots & \ddots & \ddots & \ldots & \ddots & 0 \\
& & -1 & a & b & 0 & \cdots & c \\
& & & \ddots & \ddots & \ddots & \ddots & \ddots \\
& & & & \ddots & \ddots & \ddots & 0 \\
& & & & & -1 & a & b \\
0 & & & & & & -k & t
\end{array}\right] .
$$

It is clearly showed from [2] that

$$
\operatorname{det} \mathbf{E}_{\mathbf{n}}(a, b, c, d, k, t)=\operatorname{per} \mathbf{H}_{\mathbf{n}}(a, b, c, d, k, t) .
$$

Now, we take the $n \times n$ matrix $\mathbf{H}_{\mathbf{n}}(a, b, c, 3, k, t)$ by the following form:

$$
\mathbf{H}_{\mathbf{n}}(a, b, c, 3, k, t)=\left[\begin{array}{cccccccc}
a & b & c & & & & & 0 \\
1 & a & b & c & & & & \\
& \ddots & \ddots & \ddots & \ddots & & & \\
& & 1 & a & b & c & & \\
& & & \ddots & \ddots & \ddots & \ddots & \\
& & & & \ddots & \ddots & \ddots & c \\
& & & & & 1 & a & b \\
0 & & & & & & k & t
\end{array}\right] .
$$


Then, we have

$$
\operatorname{per} \mathbf{H}_{\mathbf{n}}(a, b, c, 3, k, t)=k R_{n+1}(a, b, c, 3)-(k a-t) R_{n}(a, b, c, 3) .
$$

For example, from [9], for $a=b=c=t=k=1$ in (2.4), we have that

$$
\operatorname{per} \mathbf{H}_{\mathbf{n}}(1,1,1,3,1,1)=T_{n+1}=\operatorname{per} \mathbf{F}(n, 3),
$$

where $T_{n}$ is the $n$th tribonacci number.

For $n>1$; we define an $n \times n$ matrix $\mathbf{W}_{\mathbf{n}}(a, b, c, 3, k, t)$ as in the compact form, by the definition of $\mathbf{H}_{\mathbf{n}}(a, b, c, 3, k, t)$;

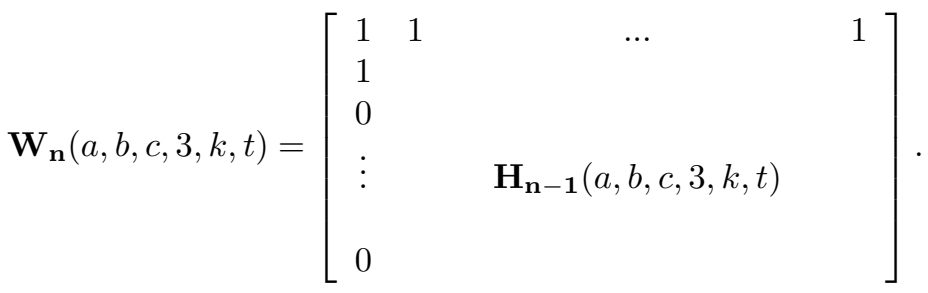

Now, we have the following theorem:

Theorem 2.2. Let $\mathbf{W}_{\mathbf{n}}(a, b, c, 3, k, t)$ be the matrix defined in (2.5). Then, for $n>2$

$$
\operatorname{per} \mathbf{W}_{\mathbf{n}}(a, b, c, 3, k, t)=k \sum_{i=1}^{n} R_{i}(a, b, c, 3)-(k a-t) \sum_{i=1}^{n} R_{i-1}(a, b, c, 3) .
$$

Proof. (Induction on $n$ ) If $n=3$, we write

$$
\begin{aligned}
& \operatorname{per} \mathbf{W}_{\mathbf{3}}(a, b, c, 3, k, t) \\
= & k+t+a t+b k=k \sum_{i=1}^{3} R_{i}(a, b, c, 3)-(k a-t) \sum_{i=1}^{3} R_{i-1}(a, b, c, 3) .
\end{aligned}
$$

Suppose that the equation holds for $n$. Then we show that the equation holds for $n+1$. From the definitions of matrices $\mathbf{H}_{\mathbf{n}}(a, b, c, 3, k, t)$ and $\mathbf{W}_{\mathbf{n}}(a, b, c, 3, k, t)$, expanding the per $\mathbf{W}_{\mathbf{n}+\mathbf{1}}(a, b, c, 3, k, t)$ with respect to the first column gives us

$$
\operatorname{per} \mathbf{W}_{\mathbf{n}+\mathbf{1}}(a, b, c, 3, k, t)=\operatorname{per} \mathbf{H}_{\mathbf{n}}(a, b, c, 3, k, t)+\operatorname{per} \mathbf{W}_{\mathbf{n}}(a, b, c, 3, k, t) .
$$

By our assumption and (2.4), we have

$$
\begin{aligned}
& \operatorname{per} \mathbf{W}_{\mathbf{n}+\mathbf{1}}(a, b, c, 3, k, t) \\
= & k R_{n+1}(a, b, c, 3)-(k a-t) R_{n}(a, b, c, 3) \\
& +k \sum_{i=1}^{n} R_{i}(a, b, c, 3)-(k a-t) \sum_{i=1}^{n} R_{i-1}(a, b, c, 3) \\
= & k \sum_{i=1}^{n+1} R_{i}(a, b, c, 3)-(k a-t) \sum_{i=1}^{n+1} R_{i-1}(a, b, c, 3) .
\end{aligned}
$$

Thus the proof is obtained. 
When $a=b=c=1$ in $(2.1)$, the sequence $\left\{R_{n}\right\}$, special case of the sequence $\left\{R_{n}(a, b, c, d)\right\}$, is defined by the recurrence

$$
R_{n}=R_{n-1}+R_{n-2}+R_{n-d}, 3 \leq d \leq n
$$

in which $R_{0}=0, R_{1}=R_{2}=\ldots=R_{d-2}=R_{d-1}=1$ and especially from (1.1), the sequence $\left\{S_{n}\right\}$ is defined by

$$
S_{n}=S_{n-1}+S_{n-2}+S_{n-d}, 3 \leq d \leq n
$$

in which $S_{0}=S_{1}=S_{2}=\ldots=S_{d-3}=0$ and $S_{d-2}=S_{d-1}=1$. For $d=3$ in $(2.6)$ and (2.7), the sequences $\left\{R_{n}\right\}$ and $\left\{S_{n}\right\}$ coincide as the tribonacci sequence.

For $n>1$, define an $n \times n$ matrix $\mathbf{Z}_{\mathbf{n}}=\left[z_{i, j}\right]$ with $z_{i+1, i}=1$ for $1 \leq i \leq n-1$, $z_{i, i}=1$ for $1 \leq i \leq n, z_{1,2}=0, z_{i, i+1}=1$ for $2 \leq i \leq n-1, z_{1, i}=1$ for $3 \leq i \leq d$, $z_{i, d+i-1}=1$ for $2 \leq i \leq n-d+1$ and 0 otherwise, i.e.

$$
\mathbf{Z}_{\mathbf{n}}=\left[\begin{array}{cccccccc}
1 & 0 & 1 & \cdots & 1 & 0 & \cdots & 0 \\
1 & 1 & 1 & 0 & \ldots & 1 & \ldots & \vdots \\
& \ddots & \ddots & \ddots & \ddots & \ldots & \ddots & 0 \\
& & 1 & 1 & 1 & 0 & \cdots & 1 \\
& & & \ddots & \ddots & \ddots & \ddots & \ddots \\
& & & & \ddots & \ddots & \ddots & 0 \\
0 & & & & & 1 & 1 & 1 \\
& & & & & & 1 & 1
\end{array}\right] .
$$

Then we give the following Theorem.

Theorem 2.3. Let $\mathbf{Z}_{\mathbf{n}}$ be the matrix defined in (2.8). Then, for $n \geq 5$ and $d \geq 3$,

$$
\operatorname{per} \mathbf{Z}_{\mathbf{n}}=R_{n+d-3}+R_{n+d-4}-R_{n+d-5}+S_{n-5}
$$

Proof. We prove this by induction on $n$. For $n=5$, we write

$$
\operatorname{per} \mathbf{Z}_{5}=2 R_{d+1}+1=R_{d+2}+R_{d+1}-R_{d}+S_{0} .
$$

The claim is true for $n=5$. Assume that the claim is true for $n-1$. Thus we show that the claim is true for $n$. Expanding the $\operatorname{per} Z_{n}$ according to the last column $d$ times, we have

$$
\operatorname{per} \mathbf{Z}_{\mathbf{n}}=\operatorname{per} \mathbf{Z}_{\mathbf{n}-\mathbf{1}}+\operatorname{per} \mathbf{Z}_{\mathbf{n}-\mathbf{2}}+\operatorname{per} \mathbf{Z}_{\mathbf{n}-\mathbf{d}} .
$$

By our assumption, we have

$$
\begin{aligned}
\operatorname{per} \mathbf{Z}_{\mathbf{n}}= & R_{n+d-4}+R_{n+d-5}-R_{n+d-6}+S_{n-6} \\
& +R_{n+d-5}+R_{n+d-6}-R_{n+d-7}+S_{n-7} \\
& +R_{n-3}+R_{n-4}-R_{n-5}+S_{n-d-5} .
\end{aligned}
$$


From the sequences $\left\{R_{n}\right\}$ and $\left\{S_{n}\right\}$, we write

$$
\operatorname{per} \mathbf{Z}_{\mathbf{n}}=R_{n+d-3}+R_{n+d-4}-R_{n+d-5}+S_{n-5}
$$

So the proof is complete.

For $n \geq 1$, define the $n \times n$ matrix $\mathbf{V}_{\mathbf{n}}^{\delta}=\left[v_{i, j}\right]$ with $v_{i+1, i}=1$ for $1 \leq i \leq n-2$, $v_{i, i}=1$ for $1 \leq i \leq n-1, v_{1, i+1}=v_{i, i+1}=(-1)^{i-\delta}$ for $1 \leq i \leq n$ and 0 otherwise, where $\delta \in\{0,1\}$. i.e.,

$$
\mathbf{V}_{\mathbf{n}}^{\delta}=\left[\begin{array}{ccccccc}
1 & (-1)^{1-\delta} & (-1)^{2-\delta} & \ldots & & (-1)^{n-2-\delta} & (-1)^{n-1-\delta} \\
1 & 1 & (-1)^{2-\delta} & 0 & \ldots & & \ldots \\
0 & \ddots & \ddots & \ddots & \ddots & \ldots & \ddots \\
& & 1 & 1 & & 0 & \cdots \\
& & & \ddots & \ddots & (-1)^{n-2-\delta} & \ddots \\
0 & & & & \ddots & 1 & (-1)^{n-1-\delta} \\
& & & & & 1 & 1
\end{array}\right] .
$$

Theorem 2.4. Let $\mathbf{V}_{\mathbf{n}}^{\delta}$ be the matrix defined in (2.9). Then, for $n \geq 1$,

$$
\operatorname{det} \mathbf{V}_{\mathbf{n}}^{\delta}=\left\{\begin{array}{cll}
(-1)^{\delta}\left(F_{\left(n+5+3(-1)^{\delta}\right) / 2}-2-(-1)^{\delta}\right) & \text { if } n \equiv 0 & (\bmod 2), \\
(-1)^{\delta}\left(F_{(n+5) / 2}-2+(-1)^{\delta}\right) & \text { if } n \equiv 1 & (\bmod 2),
\end{array}\right.
$$

where $F_{n}$ is the nth Fibonacci number.

Proof. For $n=1, \operatorname{det} \mathbf{V}_{\mathbf{1}}^{\mathbf{0}}=1=\left(F_{3}-1\right)$, $\operatorname{det} \mathbf{V}_{\mathbf{1}}^{\mathbf{1}}=1=-\left(F_{3}-3\right)$ and for $n=2$, $\operatorname{det} \mathbf{V}_{\mathbf{2}}^{\mathbf{1}}=0=-\left(F_{2}-1\right), \operatorname{det} \mathbf{V}_{\mathbf{2}}^{\mathbf{0}}=2=\left(F_{5}-3\right)$.

We show that the claim is true for $n-1$. Using expansion on the first column of $\operatorname{det} V_{n}^{1}$, we get as follows

$$
\operatorname{det} \mathbf{V}_{\mathbf{n}}^{\mathbf{1}}=\operatorname{det} \mathbf{B}_{\mathbf{n}-\mathbf{1}}^{\mathbf{0}}-\operatorname{det} \mathbf{V}_{\mathbf{n}-\mathbf{1}}^{\mathbf{0}}
$$

From (1.2) and the induction hypothesis, we have

$$
\begin{aligned}
\operatorname{det} \mathbf{V}_{\mathbf{n}}^{\mathbf{1}} & =\left\{\begin{array}{cc}
F_{n / 2}-\left(F_{(n+4) / 2}-1\right) & \text { if } n \equiv 0 \quad(\bmod 2), \\
F_{(n+3) / 2}-\left(F_{(n+7) / 2}-3\right) & \text { if } n \equiv 1 \quad(\bmod 2),
\end{array}\right. \\
& =\left\{\begin{array}{lll}
-\left(F_{((n+2) / 2}-1\right) & \text { if } n \equiv 0 \quad(\bmod 2), \\
-\left(F_{((n+5) / 2}-3\right) & \text { if } n \equiv 1 \quad(\bmod 2)
\end{array}\right.
\end{aligned}
$$

Similarly, using $\operatorname{det} \mathbf{V}_{\mathbf{n}}^{\mathbf{0}}=\operatorname{det} \mathbf{B}_{\mathbf{n}-\mathbf{1}}^{\mathbf{1}}+\operatorname{det} \mathbf{B}_{\mathbf{n}-\mathbf{2}}^{\mathbf{0}}+\operatorname{det} \mathbf{V}_{\mathbf{n}-\mathbf{2}}^{\mathbf{0}}$, the desired result is given. We have the proof. 
Theorem 2.5. For $n \geq 1$, we have

$$
\operatorname{per} \mathbf{V}_{\mathbf{n}}^{\mathbf{1}}=\left\{\begin{array}{lll}
L_{(n+2) / 2}-1 & \text { if } n \equiv 0 \quad(\bmod 2), \\
L_{(n-1) / 2}-1 & \text { if } n \equiv 1 \quad(\bmod 2),
\end{array}\right.
$$

and

$$
\operatorname{per} \mathbf{V}_{\mathbf{n}}^{\mathbf{0}}=\left\{\begin{array}{lll}
F_{(n+2) / 2}-1 & \text { if } n \equiv 0 \quad(\bmod 2), \\
F_{(n+5) / 2}-1 & \text { if } n \equiv 1 \quad(\bmod 2),
\end{array}\right.
$$

where $F_{n}$ is the nth Fibonacci number and $L_{n}$ is the nth Lucas number.

Proof. Considering $\operatorname{per} \mathbf{B}_{\mathbf{n}}^{\delta}=\left\{\begin{array}{cl}F_{(n-2+6 \delta) / 2} & \text { if } n \equiv 0(\bmod 2), \\ F_{(n+1) / 2} & \text { if } n \equiv 1(\bmod 2),\end{array}\right.$ for $\delta \in\{0,1\}$ and the equalities

$$
\operatorname{per} \mathbf{V}_{\mathbf{n}}^{\mathbf{1}}=\operatorname{per} \mathbf{B}_{\mathbf{n}-\mathbf{1}}^{\mathbf{0}}+\operatorname{per} \mathbf{V}_{\mathbf{n}-\mathbf{1}}^{\mathbf{0}} \text { and } \operatorname{per} \mathbf{V}_{\mathbf{n}}^{\mathbf{0}}=\operatorname{per} \mathbf{B}_{\mathbf{n}-\mathbf{1}}^{\mathbf{1}}-\operatorname{per} \mathbf{B}_{\mathbf{n}-\mathbf{2}}^{\mathbf{0}}+\operatorname{per} \mathbf{V}_{\mathbf{n}-\mathbf{2}}^{\mathbf{0}},
$$

we have the proof from induction on $n$.

\section{3. $\quad$ Some special cases}

In this section, we give some special cases of the above theorems:

- For $b=1, c=0$ and $d=3$, the generalized Fibonacci sequence $\left\{U_{n}(a, 1)\right\}$,

$$
\begin{aligned}
& \operatorname{per} \mathbf{H}_{\mathbf{n}}(a, 1,0,3, k, t) \\
& =\operatorname{per}\left[\begin{array}{cccccc}
a & 1 & 0 & \cdots & & \\
1 & a & 1 & 0 & & \\
& \ddots & \ddots & \ddots & \ddots & \\
& & & & & 0 \\
& & & 1 & a & 1 \\
& & & & k & t
\end{array}\right]=t U_{n}(a, 1)+k U_{n-1}(a, 1) \text {, } \\
& \operatorname{per} \mathbf{W}_{\mathbf{n}}(a, 1,0,3, k, t) \\
& =\operatorname{per}\left[\begin{array}{ccccc}
1 & 1 & 1 & \ldots & 1 \\
1 & & & & \\
0 & & & H_{n-1} & \\
\vdots & & & &
\end{array}\right] \\
& =\frac{1}{a}\left(t U \left(_{n}(a, 1)+(k+t) U\left({ }_{n-1}(a, 1)+k U_{n-2}(a, 1)+k(a-1)-t\right),\right.\right.
\end{aligned}
$$

and then,

$\operatorname{per} \mathbf{W}_{\mathbf{n}}(a, 1,0,3, k, t)=\operatorname{per} \mathbf{H}_{\mathbf{n}}(a, 1,0,3, k, t)+\operatorname{per} \mathbf{H}_{\mathbf{n}-\mathbf{1}}(a, 1,0,3, k, t)+k(a-1)+t$. 
- For $a=1, b=2, c=0$ and $d=3$ in (2.1), $\left\{J_{n}\right\}$ is the Jacobsthal sequence and for $n \geqslant 2$,

$$
\begin{aligned}
& \operatorname{per} \mathbf{H}_{\mathbf{n}}(1,2,0,3, k, t) \\
& =\operatorname{per}\left[\begin{array}{cccccc}
1 & 2 & 0 & & & \\
1 & 1 & 2 & 0 & & \\
& \ddots & \ddots & \ddots & \ddots & \cdots \\
& & & & & 0 \\
& & & 1 & 1 & 2 \\
& & & & k & t
\end{array}\right]=t J_{n}+2 k J_{n-1} .
\end{aligned}
$$

- For $a=b=k=1, c=0$ and $d=3$ in (2.1), $\left\{F_{n}\right\}$ is the Fibonacci sequence,

$$
\begin{gathered}
\operatorname{per} \mathbf{H}_{\mathbf{n}}(1,1,0,3,1, t) \\
=\operatorname{per}\left[\begin{array}{cccccc}
1 & 1 & 0 & & & \\
1 & 1 & 1 & 0 & & \\
& \ddots & \ddots & \ddots & \ddots & \ldots \\
& & & & & 0 \\
& & & 1 & 1 & 1 \\
& & & & 1 & t
\end{array}\right]=t F_{n}+F_{n-1}
\end{gathered}
$$

and

$$
\operatorname{per} \mathbf{W}_{\mathbf{n}}(1,1,0,3,1, t)=\operatorname{per}\left[\begin{array}{ccccc}
1 & 1 & 1 & \ldots & 1 \\
1 & & & & \\
0 & & & H_{n-1} & \\
\vdots & & & &
\end{array}\right]=t\left(F_{n+1}-1\right)+F_{n}
$$

- For $d=3$ in (2.6) and (2.7), $\left\{T_{n}\right\}$ is the tribonacci sequence and

$$
\operatorname{per} \mathbf{Z}_{\mathbf{n}}=\operatorname{per}\left[\begin{array}{cccccc}
1 & 0 & 1 & & & \\
1 & 1 & 1 & 1 & & \\
& \ddots & \ddots & \ddots & \ddots & \cdots \\
& & & & & 1 \\
& & & 1 & 1 & 1 \\
& & & & 1 & 1
\end{array}\right]=2 T_{n-1}+T_{n-3}+T_{n-5}
$$

\section{REF ER E N C E S}

1. R. A. Brualdi and P. M. Gibson: Convex Polyhedra of Doubly Stochastic Matrices I: Applications of the Permanents Function. J. Combin. Theory, Series A 22 (1977), $194-230$. 
2. N. D. Cahill, J. R. D'Errico, D. A. Narayan and J. Y. NArayan: Fibonacci determinants. The College Math. Journal 33 (2002), no. 3, 221-225.

3. D. Kalman: Generalized Fibonacci numbers by matrix methods. Fibonacci Quart. 20 (1982), no.1, 73-76.

4. E. KILIÇ and D. TAŞCI: On the permanents of some tridiagonal matrices with applications to the Fibonacci and Lucas numbers. Rocky Mountain J. Math. 37 (2007), no. 6, 203-219.

5. E. KILIÇ: Tribonacci sequences with certain indices and their sums. Ars Combin. 86 (2008), 13-22.

6. E. KILIÇ and D. TASCI: On families of bipartite graphs associated with sums of Fibonacci and Lucas numbers. Ars Combin. 89 (2008), 31-40.

7. E. KILIÇ and D. TAŞCI: Negatively subscripted Fibonacci and Lucas numbers and their complex factorizations. Ars Combin. 96 (2010), 275-288

8. H-C. Li: On Fibonacci-Hessenberg matrices and the Pell and Perrin numbers. Appl. Math. Comput. 218 (2012), 8353-8358.

9. H. Minc: Permanents of (0,1)-circulants. Canadian Math. Bulletin 7 (1964), 253-263.

10. J. L. RAMíREZ: Hessenberg matrices and the generalized Fibonacci-Narayana sequence. Filomat 29 (2015), no. 7, 1557-1563.

11. S. Koparal, N. ÖmÜr And C. D. ÇOlaK : Some permanents of Hessenberg matrices. Accepted in Filomat.

12. P. TrojovskÝ: On determinants of tridiagonal matrices with (-1,1)-diagonal or superdiagonal in relation to Fibonacci numbers. Global Journal of Pure and Applied Mathematics 12 (2016), no. 2, 1885-1892.

Sibel Koparal

Kocaeli University

Faculty of Art and Science

Department of Mathematics

Kocaeli, Turkey

sibel.koparal@kocaeli.edu.tr

Neşe Ömür

Kocaeli University

Faculty of Art and Science

Department of Mathematics

Kocaeli, Turkey

neseomur@kocaeli.edu.tr

Cemile Duygu Çolak

Kocaeli University

Faculty of Art and Science

Department of Mathematics

Kocaeli, Turkey

cemileduygusener@hotmail.com 